\title{
Preliminary study on the fabrication of aluminium foam through pressure assisted sintering dissolution process
}

\begin{abstract}
This paper discusses the improvement made by pressure assisted sintering dissolution process (PASDP) as opposed to the solid-state sintering dissolution process (SSSDP) which was used in producing a much improved open celled aluminium foam. The improvements leads to an aluminium foam possessing a high dense of cell wall which is attainable in a much shorter time and possesses superior mechanical properties. By varying the local sodium chloride $(\mathrm{NaCl})$ volume fractions in the sodium chloride/aluminium $(\mathrm{NaCl} / \mathrm{Al})$ compact, it is possible to fabricate aluminium foam with different relative densities. When subjected to monotonic compression mode, the fabricated aluminium foam exhibits typical stress-strain behaviour of metallic foam. The principles of Taguchi's Design of Experiments were employed to optimize processing factors for the fabrication of aluminium foam. Results were analysed based on Taguchi's signal to noise ratio $(\mathrm{S} / \mathrm{N}$ ) and analysis of variance (ANOVA) techniques in order to obtain the optimum combination of process parameter settings. Results indicate that the most notable factor influencing the fabrication of $\mathrm{Al}$ foam was the compaction at elevated temperature, followed by temperature, time and the process heating rate. The optimum processing parameters for the PASDP process were then predicted based on these results.
\end{abstract}

Keyword: Pressure assisted sintering; Open-celled; Aluminium foam; Taguchi's Design of Experiments; ANOVA 